\title{
Innovation and Creation
}

\author{
Gabriela G. Cezar \\ Assistant Professor, Animal Sciences, Human Oncology, University of Wisconsin-Madison, WI, USA
}

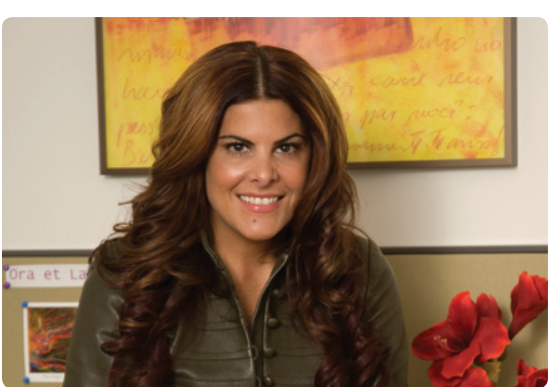

Gabriela Cezar's use of stem cells to develop diagnostic and predictive models of toxicity caught our attention. Curious to know more, BioTechniques contacted her to find out about the ambitions, character, and motivations that led to her success.

\section{How has your research affected your field?}

In stem cell research, most groups focus on therapeutic applications. I feel that my research has pushed the field toward using human embryonic stem cells (hESCs) for preclinical safety testing. Adverse drug reactions are the fourth-leading cause of death in the US, so we're trying to develop methods to help avoid serious pharmaceutical side effects while streamlining drug discovery and development.

\section{That is a daunting task. How do you approach it?}

One example is a platform that I invented where we use hESCs to predict developmental toxicity caused by pharmaceuticals and chemicals in the environment. We began by looking for a common metabolomic signature among all the drugs known to cause birth defects. I thought there would be no such signature since they act through very different mechanisms of action. But to our surprise, when we exposed hESCs to every known teratogen and then looked at the endogenous small molecules they secreted in response, we identified a number of small molecules that were only present in response to teratogenic drugs. Compared to alternative screens in the field, the developmental toxicity assay we developed was actually more predictive. We hope use of this assay will allow patients to avoid a wide range of birth defects due to chemical exposure during pregnancy.

Do you also study the mechanisms behind the toxicity?

Yes, my lab has two lines of research. One is preclinical toxicity, and the other revolves around understanding the biochemical pathways affected in neural developmental disorders, particularly autism and fetal alcohol spectrum (FAS) disorders. FAS disorders are unfortunately the largest cause of motor and mental impairment in the US. We use hESCs to create an in vitro model of human brain development and then expose the cells to the levels of alcohol known to be associated with FAS disorders. Using this methodology, we mapped out several pathways through which alcohol exerts injury during human neurodevelopment. Most importantly, we expect to generate diagnostic biomarkers, which is crucial since most women don't readily disclose alcohol consumption during pregnancy to their doctors. If we have a chemical means to identify women at risk, we can start early postnatal intervention with the babies.

We did a similar study on autism using hESCs exposed to valproate, a chemical inducer of autism, and found the pathways that were disrupted. Based on that study, we worked with the Autism Tissue Program to validate our findings in postmortem brains from autistic patients, and age-matched controls using metabolomics to determine a biochemical fingerprint of autistic brains.

What first interested you in studying stem cells and neurodevelopment disorders?

I trained as a veterinarian in Brazil and then started working on technologies for reproductive medicine, including in vitro fertilization and embryo transfer in cattle. Later, I applied for graduate school at the Roslin Institute at the University of Edinborough in Scotland; it was here I built my background in stem cell biology by studying with Ian Wilmut, who cloned Dolly the sheep in 1996. Following that, I earned my Ph.D. at the University of Wisconsin (UW)-Madison and subsequently worked in the pharmaceutical industry for about 5 years, which was very important. I'm a very pragmatic scientist; my years in the pharmaceutical industry were what actually prompted me to create better models to avoid serious side effects, thereby getting better drugs to patients.

Today I'm an assistant professor at UWMadison. I'm also cofounder of a company, Stemina Biomarker Discovery, which commercializes intellectual property that we develop at UW. Currently I'm on a leave of absence from UW-Madison and working in venture capital in Brazil.

What do you see as the biggest advantages and disadvantages of working in each of those environments?

One of the reasons I left the pharmaceutical industry was that in the industry today there seems to be less opportunity to do highly innovative work because of drug development and production costs. Companies are forced toward only commercially viable projects. In academia, the opportunity to create and innovate is an advantage that I've always loved. In venture capital, I'm putting all these elements together. In order to properly evaluate a research project or a commercial entity for investment, I need to understand the science, know what it takes to build a company, as I did, and understand the needs of the market, which I learned in the pharmaceutical industry.

Interviewed by Kristie Nybo, Ph.D. Image courtesy of the University of WisconsinMadison. 暞

BioTechniques 50:281 (May 2011)

doi $10.2144 / 000113662$

To purchase reprints of this article, contact:

biotechniques@fosterprinting.com 